AN. MED. INTERNA (Madrid) Vol. 18, N. $^{\circ} 11$, pp. 573-577, 2001

\title{
Artritis sépticas periféricas en adultos. Estudio epidemiológico en un área sanitaria gallega
}

\author{
N. GÓMEZ RODRÍGUEZ, J. IBÁÑEZ RUÁN, M. GONZÁLEZ, A. PINTADO*, \\ Y. PENELAS CORTÉS** \\ Servicios de Reumatología, *Traumatología y **Documentación Clínica. Centro Médico \\ POVISA. Vigo
}

\author{
PERIPHERAL SEPTIC ARTHRITIS IN ADULTS. EPIDEMIOLOGICAL \\ SURVEY IN A GALICIAN HEALTH DISTRICT
}

\section{RESUMEN}

Fundamento: La etiología y las manifestaciones clínico-biológicas de las artritis sépticas son bien conocidas. Sin embargo, los aspectos epidemiológicos han sido rara vez considerados. En nuestra comunidad autónoma, Galicia, apenas existen datos sobre la epidemiología de las artritis sépticas periféricas.

Objetivo: Analizar las características epidemiológicas y la etiopatogenia de las artritis sépticas periféricas en los adultos de un área sanitaria.

Material y métodos: Análisis de las historias clínicas de los pacientes correspondientes a nuestra área sanitaria del Servicio Galego de Saúde (SERGAS), con 127.000 habitantes, que fueron diagnosticados de artritis séptica en el período comprendido desde el 1 de enero de 1995 hasta el 31 de diciembre de 2000, inclusive. En todos los casos se registraron la localización, etiopatogenia y las características epidemiológicas

Resultados: Se recogieron un total de 46 artritis sépticas, 37 en articulaciones nativas y 9 en prótesis, correspondientes a 45 pacientes (24 varones y 21 mujeres), con una edad media de 57 años. La incidencia anual por cada 100.000 habitantes se redujo desde 10,2 casos en 1995 , hasta 3.1 casos en 2000 , con una media interanual de 7.2 casos $/ 10^{5}$ habitantes. Las lesiones traumáticas estuvieron implicadas en 10 casos (22\%), la mayoría en varones (8/10). Entre los factores predisponentes de los 45 pacientes destacaron la artritis reumatoide y la diabetes mellitus, ambos presentes en 5 casos (11\%), seguido de la drogadicción parenteral ( 3 casos). Staphylococcus aureus fue el microorganismo más aislado (28 pacientes, $62 \%$ ) y la rodilla fue la articulación más afectada (39\%), seguida de la cadera y tobillo, ambas implicadas en el $15 \%$.

Conclusiones: En estos 6 últimos años se ha producido una gradual reducción de la incidencia de las artritis sépticas periféricas dentro de nuestra área sanitaria, siendo destacable la trascendencia de los traumatismos como desencadenantes, tanto por inoculación directa como por la extensión intrarticular de infecciones contiguas. Los factores predisponentes generales de mayor relevancia fueron la diabetes mellitus y la artritis reumatoide. Staphylococcus aureus fue el principal agente etiológico, tanto en articulaciones nativas como protésicas.

PALABRAS CLAVE: Artritis sépticas. Epidemiología. Etiología. Patogenia.

\section{ABSTRACT}

Background: Both, clinical and biologic manifestations of septic arthritis are long been know, but few studies of its epidemiological aspects are well-documented. Literature concerning epidemiological aspects of septic arthritis remains exceptional in Galician Autonomic Community.

Objective: The aim of this investigation was to study the etiopatho genesis and epidemiological characteristic of septic arthritis in the adult population from hospital county of Servicio Galego de Saúde (SERGAS).

Material and methods: We conducted a retrospective study of all peripheral septic arthritis registered between 1 January 1995 and 31 December 2000 on adult population of our health district of SERGAS (127.000 inhabitants). The location, etiology, pathogenesis and epidemiological characteristics were obtained from medical records.

Results: The case records of 45 patients with 46 septic arthritis (37 native joints and 9 prosthetic joints) were registered. The mean age was 57 years and 24 patients were male. During the study period, the inci dence of bacterial arthritis suffered a progressive reduction to 10,2/105 inhabitants (1995) up to $3.1 / 10^{5}$ (2000) with a median of $7.2 / 10^{5}$. Ten patients (22\%), eight male and two women, had a post-traumatic septic arthritis. Major risk factors were diabetes mellitus (5 patients), rheuma toid arthritis (5 patients) and intravenous drug abuse (3 patients). Staphylococcus aureus was the principal causative agent (28 patients, $62 \%)$ and the knee was the most commonly affected joint (41\%), followed by the hip (15\%) and ankle (15\%).

Conclusions: During the past six years, the incidence of adult perip heral septic arthritis in our SERGAS health district has been gradually reduced. Trauma was a important factor in the development of septic arthritis through direct inoculation or through spread from adjacent infectious focus. The major systemic factors predisposing to joint infec tion were rheumatoid arthritis and diabetes mellitus. In both, native and prosthetic joints, Staphylococcus aureus was the most common isolated pathogen.

KEY WORDS: Septic arthritis. Epidemiology. Etiology. Pathogenesis.

Gómez Rodríguez N, Ibáñez Ruán J, González M, Pintado A, Penelas Cortés Y. Artritis sépticas periféricas en adultos. Estudio epidemiológico en un área sanitaria gallega. An Med Interna (Madrid) 2001; 18: 573-577.

Trabajo aceptado: 19 de junio de 2001

Correspondencia: Norberto Gómez Rodríguez. Servicio de Reumatología. Centro Médico POVISA. C/ Salamanca, 5 - 36211 Vigo 


\section{INTRODUCCIÓN}

Las artritis sépticas son procesos graves, inducidos por la colonización e infección de la cavidad articular por microorganismos, que provocan semiología inflamatoria local, con restricción dolorosa de los movimientos activos y pasivos. De manera inconstante, pueden presentarse escalofríos y fiebre. Biológicamente, son frecuentes la leucocitosis con neutrofilia, la elevación de la velocidad de sedimentación globular y de la proteína C reactiva, entre otros reactantes de fase inespecíficos. En la mayoría de los casos, el agente alcanza la articulación por vía hematógena, aunque también puede acceder a través de infecciones contiguas o por inoculación directa. Los retrasos en el diagnóstico y tratamiento comprometen la integridad anatómica y funcional de la articulación e incluso la vida del enfermo (1-4).

Pese al desarrollo de nuevos antibióticos y a la disponibilidad de eficaces medios de diagnóstico, las artritis sépticas constituyen todavía un problema importante, muchos de cuyos aspectos epidemiológicos no se conocen con certeza y, probablemente, varían según el área sanitaria. La tasa de incidencia de las artritis sépticas se ha estimado entre 3 y 6 casos cada 100.000 habitantes y año, si bien se considera una tendencia al aumento por influjo de una mayor esperanza de vida, incremento de las maniobras instrumentales invasivas en el aparato genitourinario y digestivo, así como por el frecuente empleo de tratamientos inmunosupresores (4).

Excepto para pediatría, nuestro centro es referencia obligada de una población de 127.000 personas. Todas las especialidades médicas se encuentran físicamente ubicadas en el hospital. Cualquier diagnóstico o proceso instrumental realizado en un paciente ingresado es registrado y codificado por el servicio de documentación clínica, lo que facilita su ulterior estudio.

Con las limitaciones que conlleva un área de población relativamente pequeña, hemos procedido a revisar, de forma retrospectiva, los casos de artritis séptica registrados en nuestra área sanitaria, desde enero de 1995 hasta el 31 de diciembre de 2000, inclusive, con especial énfasis en la etiología, mecanismos patogénicos y epidemiología.

\section{MATERIAL Y MÉTODOS}

Excepto para la atención obstétrica y pediátrica (edad $<14$ años), el centro médico POVISA es el hospital de obligada referencia de un área de población de 127.000 habitantes concertada con el Servicio Galego de Saúde (SERGAS). Excluyendo a los pacientes menores de 14 años, hemos procedido a revisar, de forma protocolizada y retrospectiva, las historias clínicas de las artritis sépticas de los pacientes pertenecientes al sector SERGAS entre el 1 de enero de 1995 y el 31 de diciembre de 2000 . No se incluyeron las artritis tuberculosas ni las espondilodiscitis.

En todos los casos se registró la edad, sexo, articulaciones afectadas, etiología, puerta de entrada (cuando pudo objetivarse), tiempo transcurrido entre el inicio de los síntomas y el diagnóstico.

El diagnóstico de artritis séptica requirió el aislamiento del microorganismo a partir líquido o de la membrana sinovial y/o la presencia de un cuadro clínico-biológico compatible asociado a dos o más hemocultivos positivos para el mismo microrganismo. La seroconversión o la positividad de las determinaciones serológicas para Brucella o Salmonella typ - hi/paratyphi, también se consideraron un criterio etiológico en presencia de un cuadro compatible.

\section{RESULTADOS}

Durante los seis años abarcados en el estudio, se recogieron un total de 46 artritis sépticas, 37 en articulaciones nativas y 9 en prótesis, correspondientes a 45 pacientes ( 24 varones y 21 mujeres). La incidencia anual por cada 100.000 habitantes se redujo desde 10,2 casos en 1995, hasta 3,1 casos en 2000, con una media interanual de 7,2 casos/10 (5) habitantes (Fig. 1). La edad media de los pacientes fue de 57 años, pero fue superior cuando se calculó en las artritis séptica sobre articulación protésica: 68 años. En conjunto, algo más de la mitad de los afectados $(55,5 \%)$ superaba los 60 años. La distribución etaria se resume en la figura 2.

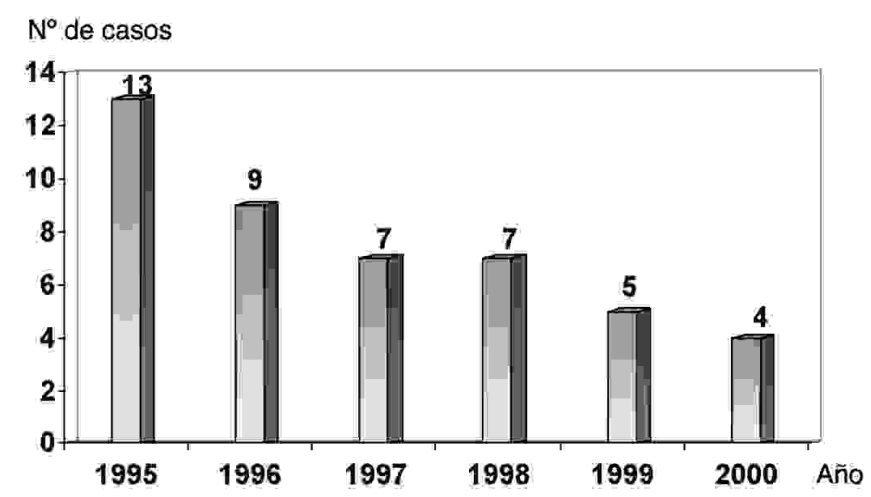

Fig. 1. Artritis sépticas periféricas en el área sanitaria (SERGASPO VISA) (127.000 habitantes).

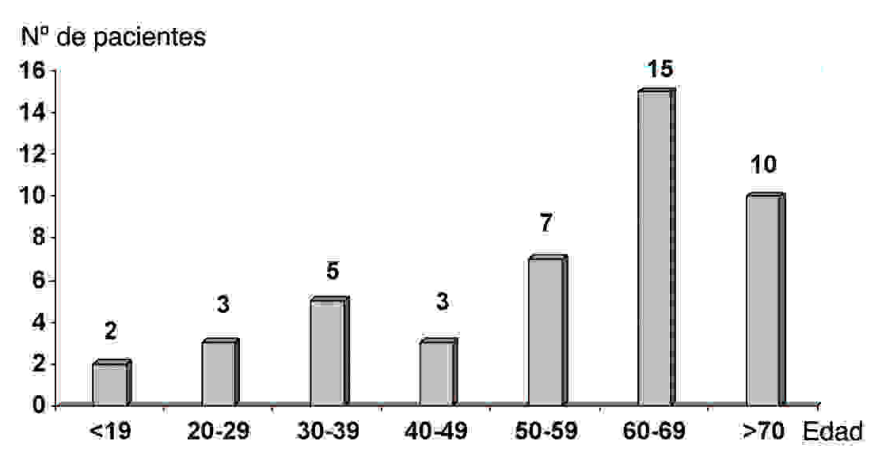

Fig. 2. Distribución etaria de las artritis sépticas periféricas en adultos del área sanitaria SERGAS-POVISA (127.000 habitantes).

En conjunto, la puerta de entrada se pudo determinar en 19 de los 45 pacientes (42\%). Las lesiones traumáticas estuvieron implicadas en 10 casos $(22 \%)$ y precedieron la artritis séptica en 8 de los 24 varones de la serie (33\%). Fueron causa de inoculación articular directa en siete enfermos, cinco de los cuales sufrieron fracturas abiertas intarticulares o yuxtarticulares, mientras que los otros dos presentaron infecciones de partes blandas contiguas como consecuencia de las heridas (Tabla I). La edad media en los casos de artritis séptica postraumática fue de 49 años. Una artritis escapulohumeral por 
TABLA I

ARTRITIS BACTERIANAS PRECEDIDAS DE TRAUM ATISM OS

\begin{tabular}{|c|c|c|c|c|}
\hline $\begin{array}{l}\text { Edad (años) } \\
\text { Sexo }\end{array}$ & Bacteria & Localización & $\begin{array}{l}\text { Tipo de } \\
\text { traumatismo }\end{array}$ & $\begin{array}{l}\text { Intervalo } \\
\text { raumatismo- síntomas) }\end{array}$ \\
\hline 36 / varón & Staphylococcus aureus & 3a IFP derecha & Penetrante yuxtarticular & 48 horas \\
\hline 54 / varón & Staphylococcus aureus & Codo izquierdo & Penetrante yuxtarticular & 28 días \\
\hline 45 / varón & Pseudomonas aeruginosa & Rodilla derecha & Fractura abierta extrarticular & 15 días \\
\hline 66 / varón & Serratia marcencens & Tobillo derecho & Fractura abierta intrarticular & $<24$ horas \\
\hline 53 / varón & Estafilococo coagulasa negativo & 4a M TF derecha & Herida punzante plantar yuxtarticular & 4 meses \\
\hline 49 / varón & Staphylococcus epidermidis & Rodilla derecha & Fractura abierta extrarticular & 30 días \\
\hline 20 / varón & Staphylococcus aureus & Rodilla derecha & Fractura abierta extrarticular & 60 años \\
\hline 36 / varón & $\begin{array}{l}\text { Streptococcus mitis + } \\
\text { Pseudomonas aeruginosa }\end{array}$ & Tobillo izquierdo & $\begin{array}{l}\text { Fractura abierta intrarticular + } \\
\text { polifracturas y abrasiones }\end{array}$ & 7 días \\
\hline 62 / mujer & Staphylococcus aureus & Cadera derecha & $\begin{array}{l}\text { Múltiples heridas incisocontusas } \\
\text { en extremidades inferiores }\end{array}$ & 7 días \\
\hline 78 / mujer & Streptococcus agalactiae & Tobillo izquierdo & Herida penetrante yuxtarticular & 120 días \\
\hline
\end{tabular}

IFP (interfalángica proximal) M TF (metatarsofalángica)

Staphylococcus aureus estuvo precedida en 14 días por una infiltración, aunque el número total de infiltraciones realizadas durante el periodo de estudio no pudo establecerse.

Entre los factores predisponentes de los 45 pacientes destacaron la artritis reumatoide y la diabetes mellitus, ambos presentes en 5 casos $(11 \%)$, seguido de la drogadicción parenteral (3 casos) y, finalmente, cáncer y síndrome de Sjögren, ambos en un enfermo (Tabla II).

Todos los casos fueron confirmados por cultivo de líquido o de la membrana sinovial. Staphylococcus aureus fue el microorganismo más aislado (28 pacientes, 62\%), seguido de los estafilococos coagulasa negativos (4 pacientes, $9 \%$ ). Se identificaron dos microorganismos en el líquido o en la membrana sinovial de dos pacientes, uno de ellos con artritis por Streptococcus mitis y Pseudomonas aeruginosa tras una fractura abierta del tobillo izquierdo. El otro enfermo, diabético, presentó una artritis escápulohumeral derecha con aislamiento de Staphylococcus aureus y Proteus mirabilis, proceso precedido por la colocación de un by-pass fémoro-poplíteo. Un paciente, que dos semanas antes había ingresado por paludismo, presentó una endocarditis y una artritis séptica de su cadera izquierda (nativa) por Enterococcus faecalis, cuyo origen fue probablemente nosocomial. Por último, se aisló Sal -

\section{TABLA II}

\section{FACTO RES PREDISPO NENTES GENERALES EN LAS ARTRITIS SÉPTICAS (45 PACIENTES)}

\begin{tabular}{lcc}
\hline Factores predisponentes & Pacientes & $\%(\mathrm{n} / 45 \times 100)$ \\
\hline Diabetes & 5 & $11 \%$ \\
Artritis reumatoide & 5 & $11 \%$ \\
ADVP + SIDA* & 3 & $7 \%$ \\
Cáncer & 1 & $2 \%$ \\
Otras** & 2 & $4 \%$
\end{tabular}

ADVP*: Adicción a drogas por vía parenteral. SIDA: síndrome de inmunodeficiencia adquirido. O tras**: síndrome de Sjögren (1) y hepatitis crónica C (1). monella $s p$ en las muestras de la sinovial (cirugía artroscópica) del hombro derecho de un enfermo cuya sintomatología se había iniciado 40 días antes y que se había precedido de enterocolitis. El espectro etiológico de las artritis sépticas se resumen en la tabla III.

TABLA III

ESPECTRO ETIOLÓGICO DE LAS ARTRITIS SÉPTICAS

\begin{tabular}{lcc}
\hline M icroorganismos aislados & Nativas & Protésicas \\
\hline Staphylococcus aureus & 26 & 5 \\
Estafilococos coagulasa negativos & 2 & 2 \\
Streptococcus agalactiae & 2 & 0 \\
Streptococcus milleri & 1 & 0 \\
Streptococcus pyogenes $g^{0}$ A & 1 & 0 \\
Streptococcus mitis $(*)$ & 1 & 0 \\
Pseudomonas aeruginosa $(*)$ & 2 & 0 \\
Salmonella sp & 1 & 0 \\
E. coli & 1 & 1 \\
Serratia marcencens & 1 & 1 \\
Streptococcus faecalis & 1 & 0 \\
\hline
\end{tabular}

(*) En el hombro derecho de un paciente diabético se aisló Proteus mira bilis $+S$ aureus.

En el tobillo derecho de un politraumatizado se aisló S. mitis y P.aeruginosa.

La rodilla fue la articulación más afectada (19 casos, $41 \%$ ), seguida de la cadera y tobillo, ambas implicadas en 7 pacientes $(15 \%)$. Tanto las metatarsofalángicas como los hombros estuvieron involucrados en 4 pacientes (9\%). Otras localizaciones fueron la articulación esternoclavicular derecha, codo izquierdo, $1^{\mathrm{a}}$ metacarpofalángica izquierda, $3^{\mathrm{a}}$ interfalángica proximal derecha y radiocarpiana derecha, todas ellas en un paciente (Fig. 3). 


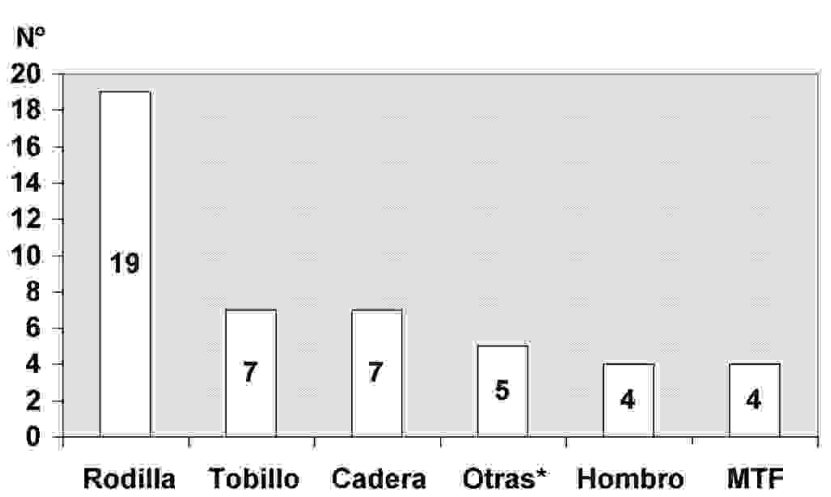

Fig. 3. Articulaciones afectadas en los 45 pacientes con artritis séptica periférica del sector sanitario SERGAS-POVISA (127.000 habitantes).

O tras*: esternoclavicular derecha, codo izquierdo, 1a metacarpofalángica izquierda, 3 a interfalángica proximal derecha, radiocarpiana derecha. Cada una de estas articulaciones estuvieron implicadas en sólo un paciente.

En el periodo de estudio se efectuaron 1.069 artroplastias de cadera y se colocaron 592 prótesis totales o parciales de rodilla. Las artritis sépticas sobre articulación protésica representaron $2 / 1.069(0,18 \%)$ para las caderas y $7 / 592(1,2 \%)$ para las rodillas.

\section{DISCUSIÓN}

Este estudio descriptivo y retrospectivo se ha realizado a lo largo de seis años, período que nos parece bastante representativo. No obstante, hemos de reconocer las limitaciones generadas por el reducido número de casos registrados y por la posibilidad de que algún enfermo del Sector Sanitario SERGAS asignado a POVISA, bajo la cobertura adicional de una compañía privada de seguros de salud, haya buscado asistencia en otro hospital. Con todo, aunque los datos epidemiológicos reseñados no puedan extrapolarse a otras áreas gallegas, creemos que reflejan la realidad de las artritis sépticas periféricas en los adultos de nuestro sector sanitario.

Fueron hallazgos comunes con la mayoría de las series, el predominio masculino, el incremento de la frecuencia con la edad y la participación mayoritaria de Staphylococcus aureus $(2,4)$. Sin embargo, frente a lo cabría esperar, la incidencia anual por cada 100.000 habitantes, muy elevada en 1995 (10,2 casos) se redujo hasta 3.1 casos en el último año del estudio. Aún así, la media interanual quedó establecida en 7,2 casos $/ 10^{5}$ habitantes, cifra sensiblemente elevada cuando se compara con la registrada por otros autores (4-8), sobre todo si tenemos en cuenta que no se ha incluido la población pediátrica. A este respecto, en la serie de Kaandorp et al, recogida de diversos distritos sanitarios de Amsterdam y que englobaba tanto a adultos como a niños, la incidencia de artritis séptica se estimaba en 5,7 casos por cada 100.000 habitantes y año (8).

El predominio masculino es prácticamente constante en todas las series, incluida la que presentamos, y, en general, la mayoría de los casos son monoarticulares (6-11). El 55\% de nuestros pacientes tenían más de 60 años, lo que pone de manifiesto el incremento de la prevalencia de este proceso con la edad $(4,8)$. Su incidencia sobre articulaciones protésicas $(0,18 \%$ en caderas y $1,2 \%$ en rodilla) fue inferior a lo objeti- vado en otras series $(8,12-14)$. Estos datos deben interpretarse con cautela, puesto que la ratio de infecciones está condicionada, entre otros factores, por el tiempo de seguimiento, las enfermedades de base y el uso de inmunosupresores. La presencia de material de osteosíntesis facilita la adhesión bacteriana y, por lo tanto, incrementa la probabilidad de infección articular (14), al tiempo que el propio acto quirúrgico permite el acceso directo de agentes infecciosos.

Las lesiones traumáticas estuvieron implicadas en el $22 \%$ de los casos, sobre todo en varones, lo que traduce la importancia de los accidentes como causa directa o indirecta de artritis séptica en nuestro medio. La cirugía articular constituyó el segundo origen más frecuente de la infección articular directa $(20 \%)$. De hecho, las artroplastias e instrumentaciones constituyen la causa más frecuente de artritis infecciosa por inoculación directa (8). La artritis reumatoide y la diabetes mellitus, factores predisponentes bien documentados en la literatura $(1,3,4)$, fueron los más significativos en nuestra serie, seguidas de la drogadicción y las neoplasias. En ninguna de las historias de nuestros pacientes se consignaba el alcoholismo, cuya prevalencia en nuestro medio es notable, ya que más del $10 \%$ de población puede considerarse bebedora excesiva. Dos de los enfermos, ambos adictos a drogas por vía parenteral, sufrían infección por el virus de inmunodeficiencia humana tipo I (VIH), proceso que facilita infecciones por otros microorganismos, incluidos los oportunistas. Sin embargo, en conjunto, las infecciones del aparato locomotor en pacientes con síndrome de inmunodeficiencia adquirida tienen una baja prevalencia $(15,16)$, aun cuando las más habituales sean precisamente las artritis sépticas (17).

La mayoría de las artritis sépticas son producidas por un solo microorganismo y Staphylococcus aureus es el más aislado, tanto en articulaciones nativas como en las protésicas $(8,15,16)$. Aunque en la mayoría de las series anglosajonas la Neisseria gonorroheae es uno de los principales agentes de artritis séptica, alcanzando el 50\% del total, entre nuestros casos no se aisló esta bacteria, la cual, a diferencia de otras gramnegativas, ha ido perdiendo protagonismo en los últimos años. En España, como otros países europeos, se ha estimado que las artritis gonocócicas suponen menos del $11 \%$ del total (9), e incluso entre los usuarios de drogas por vía parenteral, provoca menos del 3\% de las infecciones osteoarticulares (15), porcentaje similar al registrado en algunas áreas de Gran Bretaña (18). Los restantes microorganismos gramnegativos (29\% en nuestra serie) podrían estar adquiriendo una mayor relevancia, no sólo por el incremento de la esperanza de vida y la cada vez mayor frecuencia de maniobras instrumentales en el tracto digestivo y genitourinario, sino también por la aplicación de tratamientos inmunosupresores en enfermedades neoplásicas y autoinmunes. Los enterococos se han comunicado en raras ocasiones como etiología de artritis séptica. En nuestra serie recogimos un paciente con endocarditis e infección monomicrobiana de su cadera izquierda (nativa) por Enterococcus faecalis. La mayor parte de las artritis en las que esta bacteria estuvo implicada han sido comunicadas en articulaciones protésicas y, en un tercio de los casos, fueron polimicrobianas (19). Los estafilococos coagulasa negativos, especialmente Staphylococcus epidermidis, se aislan fundamentalmente en las infecciones sobre prótesis articulares durante el periodo postquirúrgico precoz. En nuestra serie estos agentes fueron los causante de la infección de dos prótesis de rodilla correspondientes a dos pacientes. 
Las artritis sépticas predominan en las articulaciones de carga (60-80\% casos), ocupando la rodilla el primer lugar, seguida de la cadera y, con frecuencia variable, otras articulaciones como hombro y tobillo $(1,9,20,21)$. La cadera y el hombro son localizaciones especialmente problemáticas por la necesidad de desbridamiento quirúrgico abierto o artroscópico y las secuelas funcionales. Las artritis sépticas escápulohumerales representaban aproximadamente el 3\% del total en las series amplias, pero su frecuencia podría estar aumentando. En nuestro estudio, supusieron el $9 \%$ del total y en una reciente revisión (11), alcanzaban el $12 \%$.

\section{Bibliografía}

1. Ibáñez AE, González M. Artritis séptica. Med Clin (Barc) 1986; 87 : 861-866.

2. Goldenberg D L, Reed J I. Bacterial arthritis. N Engl J Med 1985; 312: 764-771.

3. Smith J W. Infectious arthritis. Infect Dis North Am 1990; 4: 523538

4. Bouza E, Carreño L. Artritis de articulaciones periféricas por microorganismos piógenos. En:Pascual E, Rodríguez Valverde V, Carbonell J, Gómez-Reino J J, Eds. Tratado de Reumatología. Tomo I. Madrid, ARAN Ediciones SA, 1998; p: 1267-1280.

5. Lidgren L, Lindberg L. Twenty-nine cases of bacterial arthritis. Acta Orthop Scand 1973; 44: 263-269.

6. Mayers A R. Septic arthritis caused by bacteria. En, Textbook of Rheumatology 1rd, ed. Kelley W N, Harris E D, Ruddy S, Sledge C D, eds. Philadelphia, W B Saunders Company 1981, p: 1551-1572.

7. Kaandorp C J E, Dinant H J, van Schaardenburg D, van der Laar MAFJ. Septic arthritis in Amsterdam: Potencial targets for prevention. Br J Rheumatol 1994; 34 (supp 1): 216.

8. Kaandorf C J E, Dinant H J, Van de Laar M A F J, Moens H J B, Prins A P A, Dijkmans B A C. Incidence and sources of native and prosthetic joint infection: a community based prospective survey. Ann Rheum Dis 1997; 56: 470-475.

9. Rozadilla A, Nolla J M, Mateo L, del Blanco J, Valverde J, Roig D. Artritis séptica inducida por gérmenes piógenos en pacientes sin adicción a drogas por vía parenteral. Análisis de 44 casos. Med Clin (Barc) 1992; 98: 527-530.

10. Studahl M, Bergman B, Kälebo P, Lindberg J. Septic arthritis of the knee: a 10-year review and long-term follow-up using a new scoring system. Scand J Infect Dis 1994; 26: 85-93.

11. Lossos I S, Yossepowitch O, Kandel L, Yardeni D, Arber N. Septic arthritis of the glenohumeral joint. A report of 11 cases and review of the literature. Medicine (Balt) 1998; 77: 177-187.

12. Bengston S, Knutson K. The infected knee arthroplasty: a 6-year follow-up of 357 cases. Acta Orthop Scand 1991; 62: 301-311.
Las características clínicas, biológicas y radiológicas de las artritis sépticas no constituyeron objeto de nuestro estudio, aparte de la abundante información sobre estos aspectos que puede recabarse en la literatura nacional $(1,4,9,21,22)$, series extranjeras $(8,10,23)$ y textos de reumatología $(4,24)$.

En resumen, en nuestra área sanitaria, creemos necesario destacar la gradual reducción de la incidencia de las artritis sépticas durante los últimos 6 años, el relevante papel de los traumatismos como factores desencadenantes $\mathrm{y}$, entre los factores predisponentes, la importancia de la diabetes mellitus y de la artritis reumatoide.

13. Wymenga A B, van Horn J R, Theeuwes A, Muytjens $H$, Slooff $T$ J. Perioperative factors associated with septic arthritis after arthroplasty. Prospective multicentric study of 362 Knee and 2.651 hip operations. Acta Orthop Scand 1992; 63: 665-671.

14. Berbari E F, Hanssen A D, Duffy M C, Steckelberg J M, Ilstrup D M, Harmsen W S, Osmon D R. Risk factors for proshetic joint infection: case-control study. C I D 1998; 27: 1.247-1.254.

15. Grupo de Trabajo para el Estudio de Infecciones en Drogadictos. Estudio epidemiológico de las complicaciones infecciosas en adictos a drogas por vía parenteral en España: análisis de 11.645 casos (1977-1988). Enferm Infecc Microbiol Clin 1990; 8: 514-519.

16. Ike R W. Bacterial arthritis. Curr Opin Rheumatol 1998; 10: 330-334.

17. Vassilopoulos D, Calasani P, Jurado R L, Workowski K, Agudelo C A. Musculoskeletal infections in patients with human immunodeficiency virus infection. Medicine (Balt) 1997, 76: 284-294.

18. Ryan M J, Kavanagh R, Wall P G, Hazleman B L. Bacterial joint infections in England and Wales: analysis of bacterial isolates over a four year period. Br J Rheumatol 1997; 36: 370-373.

19. Raymond N J, Henry , Workowski K A. Enterococcal arthritis: case report and review. C I D 1995; 21: 516-522.

20. Gomis M, Sánchez B, Ledesma F, Martínez D. Artritis infecciosas: perfil etiológico actual. Rev Clin Esp 1996; 196: 33-43.

21. Pascual A, Coret V, Ureña I, Moya R, Miralles F, González V et al. Artritis séptica: descripción de una serie de casos. Rev Esp Reumatol 1999; 26: 161 (abstract).

22. Moreno M, López F J, González C M, Bascones M, Turrion A I, Montilla C M et al. Artritis sépticas: veinte años de experiencia en un hospital general. Rev Esp Reumatol 2000; 197 (abstract).

23. Kaandorp C J E, Krijnen P, Moens H J B, Habbema J D F, Vanschaardenburg D. The outcome of bacterial arthritis: A prospective community-based study. Arthritis Rheum 1997; 40: 884-892.

24. Goldenberg D L. Bacterial arthritis. In: Kelley W N, Harris E D Jr, Ruddy S, Sledge C B. Eds. Textbook of Rheumatology, 5th Edition. Philadelphia. W.B. Saunders Company 1997; p: 1.435-1.449 